\title{
Rôle inattendu du facteur de transcription NF-E2 dans la maturation terminale du mégacaryocyte et la production des plaquettes
}

L'isolement récent du facteur humoral réglant physiologiquement la production plaquettaire, la thrombopoiétine, a mis la lignée mégacaryocytaire à l'honneur ( $\mathrm{m} / \mathrm{s} n^{\circ} 8-9$, vol 10, p. 874). De nombreux travaux menés in vivo et in vitro ont démontré le rôle crucial de cette cytokine dans les processus de prolifération, de ploïdisation, de maturation cytoplasmique et de production des plaquettes. Les plaquettes proviennent d'un processus coordonné de maturation du cytoplasme du mégacaryocyte. L'hypothèse actuellement la plus probable est que le cytoplasme du mégacaryocyte mûr s'allonge, formant des pseudopodes. Le long de ces pseudopodes apparaissent des constrictions qui délimitent les futures plaquettes. La formation de ces pseudopodes est finement réglée et plusieurs régulateurs ayant une action positive ou négative sont étudiés. Une autre hypothèse, que favorisent les auteurs, est que les territoires plaquettaires se délimitent à l'intérieur du cytoplasme du mégacaryocyte. Quoi qu'il en soit, ces études descriptives ne permettent pas d'identifier les gènes impliqués dans ces mécanismes. Un travail réalisé par l'équipe de Stuart Orkin, en collaboration avec d'autres laboratoires américains, ouvre une piste prometteuse dans l'identification des gènes cibles impliqués dans la formation des plaquettes [1].

Afin de définir le rôle physiologique $\mathrm{du}$ facteur de transcription NF-E2, ces auteurs ont suivi la stratégie d'inactivation de gène par recombinaison homologue chez la souris. Le facteur de transcription NF-E2 a été découvert lors de l'étude du promoteur érythrocytaire du gène de la porphobilinogène désaminase [2]. Son rôle important dans le régulation des gènes de $\beta$-globine a ensuite été mis en évidence $\left(\mathrm{m} / \mathrm{s} n^{\circ} 3\right.$, vol. $8, p$. 257), suggérant un rôle de NF-E2 dans la régulation de gènes spécifiques de la lignée érythroïde. NF-E2 est un complexe hétérodimérique constitué d'une protéine de $45 \mathrm{kDa}$ (p45) apparentée à Jun/AP-l, qui n'est exprimée que dans les tissus hématopoïétiques, et d'une protéine de $18 \mathrm{kDa}$ (pl8) d'expression ubiquitaire, apparentée à la famille des protéines Maf. L'inactivation génique a porté sur la sous-unité p45, qui est coexprimée avec GATA-1 dans les lignées érythrocytaire, mégacaryocytaire et mastocytaire [3, 4].

Contre toute prédiction, les souris homozygotes $p 45 N F-E 2$-/- ont une érythropoïèse embryonnaire et fœtale apparemment normale. En revanche, et de manière inattendue, $90 \%$ des souriceaux meurent à la naissance d'hémorragies liées à une absence totale de plaquettes $(\mathrm{m} / \mathrm{s}$ $n^{\circ} 9$, vol. 11, p. 1355). Les auteurs mentionnent que moins de $10 \%$ des animaux homozygotes survivent jusqu'à un âge adulte, mais ne donnent pas d'indication sur les mécanismes hémostatiques permettant leur survie. Le nombre et la morphologie des granulocytes et des lymphocytes sont normaux dans le sang et dans la moelle osseuse. Au niveau de l'érythropoïèse, une anémie modérée, une microcytose et une teneur rédui- te en hémoglobine sont observées, ces anomalies étant probablement la conséquence des saignements. Dans la moelle osseuse et la rate, de très nombreux mégacaryocytes (augmentation de 2 à 4 fois par rapport aux animaux normaux ou hétérozygotes) et de haute ploïdie sont présents. Ces cellules expriment les ARN messagers du facteur PF4 et de la glycoprotéine plaquettaire GpIIb. Les études en microscopie électronique révèlent une désorganisation du cytoplasme des mégacaryocytes avec une augmentation des membranes de démarcation et une diminution marquée des granules $\alpha$. D'après les auteurs, la trombopénie serait liée à l'absence de délimitation des territoires plaquettaires. Les noyaux des mégacaryocytes sont également anormaux avec une hyperlobulation et une exclusion de fragments nucléaires. Les mégacaryocytes des animaux homozygotes expriment le récepteur de la thrombopoiétine (c-Mpl). De plus, ce récepteur est fonctionnel puisque, lorsque de la thrombopoiétine (TPO) est injectée aux souris, le nombre de mégacaryocytes augmente de manière spectaculaire dans les tissus cibles sans toutefois produire de plaquettes circulantes. Une hypothèse, qui a été exclue, pouvait être que le sérum des souris homozygotes contenait une activité capable de détruire les plaquettes. Aucune diminution du nombre de plaquettes n'est observée chez des souris normales recevant du sérum de souris p45NF-E2-/-. Une autre hypothèse pouvait être un défaut de production 
de TPO. Une analyse réalisée par RTPCR montre des transcrits dans le foie fotal. Cependant, aucune activité biologique n'est retrouvée dans le sérum des animaux thrombopéniques. Cette observation appelle un commentaire puisque de très nombreux travaux montrent une corrélation inverse stricte entre le nombre de plaquettes et le niveau détectable de TPO dans le sérum. Une explication simple pourrait être que la TPO est produite mais rapidement fixée sur les récepteurs présents au niveau des mégacaryocytes qui, chez ces animaux, sont en nombre augmenté. Ces travaux révèlent le rôle critique de p45NF-E2 dans la régulation de gènes cibles jouant un rôle clé dans la maturation terminale des mégacaryocytes et la formation des plaquettes. Ce modèle apporte un outil extrêmement puissant pour identifier ces gènes et nul doute que leur caractérisation sera donnée prochainement. Ces études essentielles, qui n'avaient pu être entreprises faute de modèle, permettront de décrypter les bases moléculaires de la production plaquettaire.
I. Shivdasani RA, Rosenblatt MF, Zucker-Franflin D, Jackson CW, Hunt P, Saris CJM, Orkin SH. Transcription factor NF-E2 is required for platelet formation independent of the actions of thrombopoietin/MGDF in megakaryocyte development. Ciell $1995 ; 81$ : 695-4.

2. Mignotte V, Watt L, deBoer E, Grosveld F, Romeo PH. Two tissue-specific factors bind the erythroid promoter of the human porphobilinogen deaminase gene. Nucleic Acids Res 1989; 17 : $37-4$.

3. Roméo PH, Prandini MH, Joulin V, Mignotte V, Prenant M, Vainchenker W, Maruerie G, Uzan (i. Megakaryocytic and erythrocytic lineages share specific transcription factors. Nature 1990; 344 : 447-9.

4. Andrews NC, Erjument-Bromage $\mathrm{H}$, Davidson F.W. MB, Tempst P, Orkin SH. Erythroid transcription F.W. factor NF-E2 is a haematopoietic-specific basicW.V. leucine zipper protein. Nature $1993 ; 363: 722-8$. 